\title{
Surgical Treatment for Ipsilateral Fractures of Femoral Neck and Shaft
}

\author{
F. Khallaf M. Al-Mosalamy M. Al-Akkad H. Hantira \\ Department of Orthopedics, Al-Jahra Hospital, Ministry of Health, Kuwait
}

\author{
Key Words \\ Ipsilateral femoral neck and shaft fractures . \\ Plate fixation - Dynamic hip screw · Nonunion • \\ Osteonecrosis
}

\begin{abstract}
Objectives: To review the primary and the final results of surgical management of ipsilateral femoral neck and shaft fractures. Subjects and Methods: Seventeen patients ( 2 female, 15 male, average age 37 years, range 20-60) with ipsilateral femoral neck and shaft fractures were treated by compression plate fixation for the femoral shaft fractures and dynamic hip screw or cannulated cancellous screw fixation for the neck fractures in Al-Razi and Al-Jahra Hospitals, Kuwait, from March 1996 to June 2002. The clinical and radiological primary and final results were analyzed retrospectively. Results: The average follow-up for the primary outcomes was 3.6 years (range 2-6 years). 100\% union of the femoral neck fractures, $0 \%$ osteonecrosis of the femoral head and $77 \%$ union of the shaft fractures were achieved. Complications of femoral shaft fractures, nonunion and metal failure, occurred in $4(23 \%)$ patients and postsurgical infection occurred in 1 patient. At the final follow-up, 15 (88\%) patients achieved good clinical outcome and $2(12 \%)$ had fair outcome. Conclusion: Our results show that com-
\end{abstract}

pression plate fixation for the femoral shaft fracture and dynamic hip screw or cannulated screw fixation for the neck fracture in combination injury is a reliable and adequate technique.

Copyright (C) 2005 S. Karger AG, Basel

\section{Introduction}

Ipsilateral femoral neck and shaft fractures are uncommon injuries $[1,2]$. They occur exclusively in high velocity injuries like road traffic accidents and falling from a height. They are commonly associated with other skeletal injuries, chest injuries and abdominal injuries [3, 4]. Many literature reports described a specific pattern of femoral neck fracture, which in most instances is a vertical and nondisplaced or minimally displaced fracture that is often missed [5-8]. Numerous protocols have been recommended for the treatment of this combination injury. An evidence-based orthopedic trauma meta-analysis of the literature reports showed different surgical treatment options, which include: (a) antegrade interlocking femoral nailing of the shaft with cannulated cancellous screws placed anteriorly to the nail for fixation of the neck; (b) reconstruction intramedullary nailing using proximal interlocking screws that pass through the proximal nail segment, across the femoral neck fracture and into the 
Fig. 1. Vertical shear midcervical intracapsular fracture Garden III is the most common neck fracture in our series.

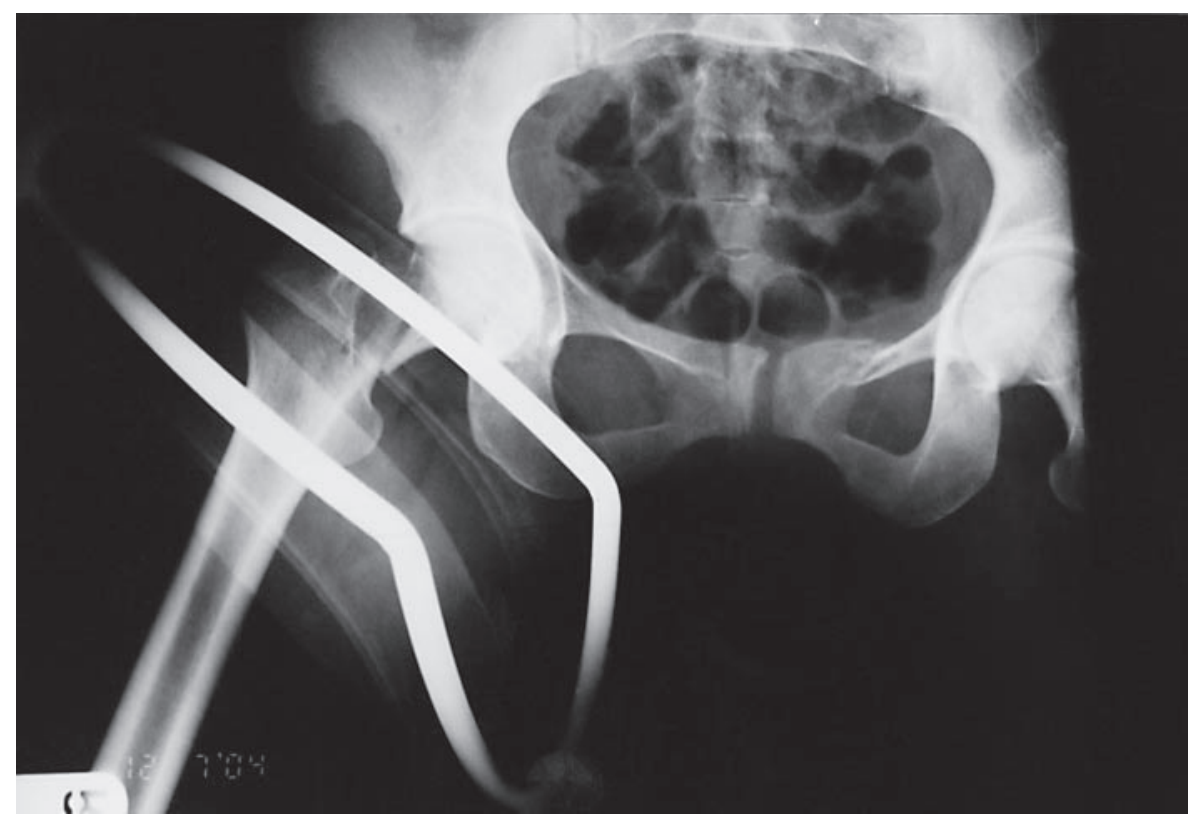

femoral head (with and without the use of additional cannulated cancellous lag screws to augment the neck fixation); (c) various plate combinations including dynamic hip screw (DHS) with long side plate, short side plate for the neck and separate dynamic compression plate (DCP) for the shaft and cannulated cancellous screws for the neck and a plate for the shaft, and (d) retrograde intramedullary nailing for shaft fixation with cancellous lag screws placed superiorly to the tip of the nail for neck stabilization.

All these techniques have achieved varying degrees of success, with the occurrence of femoral shaft nonunion in $2-10 \%$ and complications involving the femoral neck reported as high as $25 \%[7,9,10]$. A standard model of fixation of these combination injuries has not yet been reached or recommended.

This report is a review of the results of surgery of this complex injury, plating the femoral shaft and fixation of the femoral neck by DHS or cannulated lag screws. The pattern of complications, particularly post-traumatic osteonecrosis of the femoral head and nonunion of femoral neck and shaft fractures, is documented.

\section{Subjects and Methods}

Seventeen patients with ipsilateral femoral neck and shaft fractures treated surgically in Al-Razi and Al-Jahra Hospitals in the period from March 1996 to June 2002 formed the basis of this report.

Ipsilateral Fractures of Femoral Neck and Shaft

\section{Subjects}

The study was a nonrandomized retrospective analysis of 17 adult patients ( 15 male and 2 female, average age 37 years, range 20-60) involved in high-energy trauma that resulted in ipsilateral femoral neck fractures. Sixteen patients were involved in road traffic accidents while the remaining patient had a history of falling from a height. Twelve of the patients had Garden III displaced intracapsular midcervical shear femoral neck fractures as shown in figure 1. The remaining 5 had Garden III displaced basal neck fracture $[11,12]$. Twelve patients had midshaft displaced femoral fractures while the other 5 had displaced femoral shaft fractures at the junction between the middle and lower third. Two of the femoral shaft fractures were open Gustilo-Andersen grade II [13]. All the diaphyseal femoral fractures were comminuted. Six patients were type II, 8 patients type III and 3 patients type IV according to the Winquist-Hansen classification [14, 15]. Two of the patients had associated skeletal injuries, 1 had closed comminuted fracture of the tibia and fibula of the contralateral leg, and the other patient had moderate wedge fracture of D12 and L1 vertebrae with no neurological deficits. Three patients had other nonorthopedic associated injuries, which included: chest injury with hemothorax of the contralateral side of the ipsilateral neck and shaft femoral fractures. The other 2 had head injury with 1 having Lee-Fort type II facial bone fracture. No fracture of the femoral neck in our group of patients was diagnosed initially or retrospectively. All the patients were treated operatively by reduction and fixation of the neck fractures by DHS or cannulated screws and fixation of the femoral shaft fractures by DCP. The average period between admission and surgery was 3 days and the range was $2-6$ days. Results of surgical management of the femoral neck and shaft fractures were analyzed separately. Patients were excluded if they failed to survive long enough to have their fractures definitively managed and adequately followed up. Patients whose femoral injuries were treated, either in whole or in part, at other institutions rather than those previously mentioned were also excluded. The patients' medical records, 
Fig. 2 a, b. Fixation of the femoral neck fracture by cannulated screws and the femoral shaft fracture by long broad DCP, immediately postoperatively.
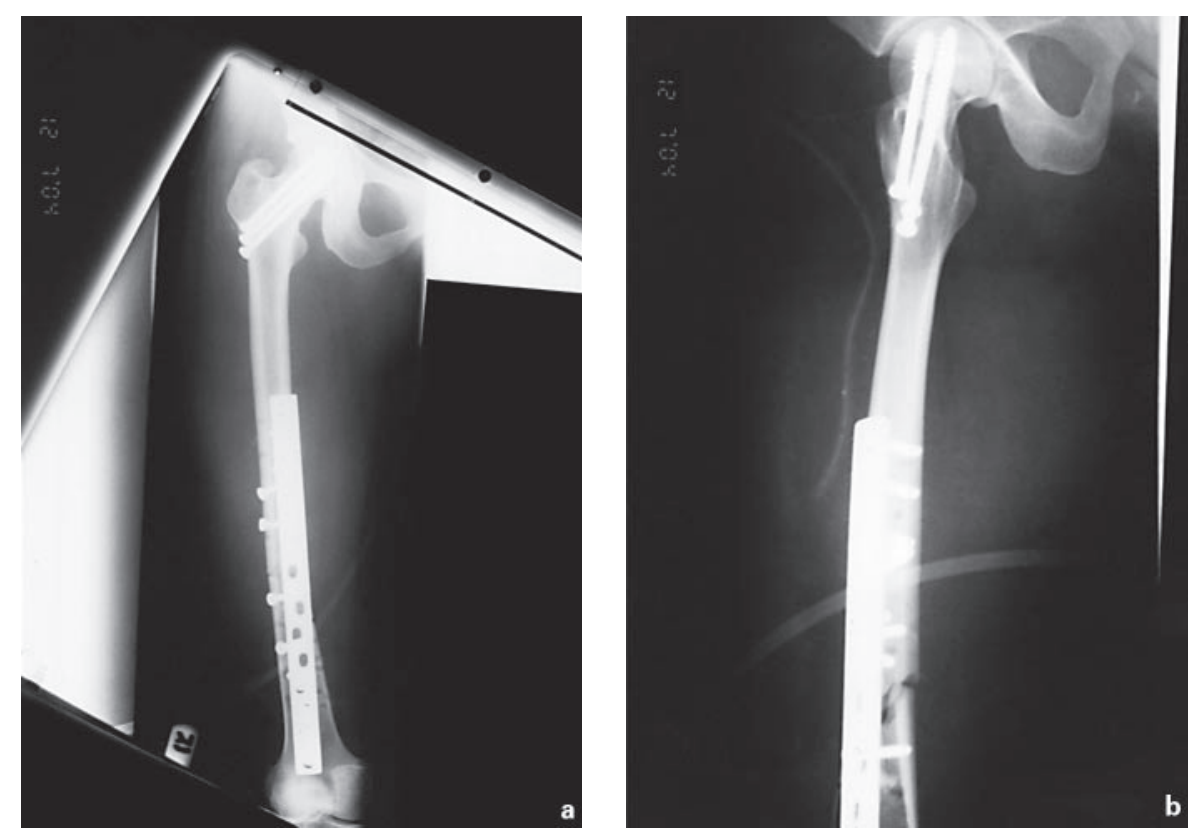

operative reports and radiographs were reviewed to determine their demographic data, date and mechanism of injury, the presence and classification of open fractures, classification and displacement of sustained fractures, timing of stabilization, methods of reduction and types of stabilizing implants.

\section{Surgical Treatment}

The same surgical technique and details were followed in all but in 2 patients. First, with the patient in the semilateral position and through a lateral approach, the diaphyseal femoral facture was addressed. In 13 patients, a 12-hole DCP on the lateral aspect was used to fix the diaphyseal fracture and a 14-hole DCP in the remaining 4 patients. At least a 4- to 6-screw plate fixation to the femoral shaft proximal and distal to the fracture comminution was inserted. After open reduction, femoral diaphyseal fracture stabilization and insertion of a Hemovac drain, the wound was closed in layers. Then, the patient was put in supine position on the orthopedic table and a closed reduction for the neck fracture by traction, abduction and internal rotation was performed. The reduction was performed according to Garden's [11, 12] alignment index. The index that includes the anteroposterior view of the hip, the angle formed by the central axis of the medial head trabeculae and the medial cortex of the femoral shaft and the angle between the central axis of the femoral head and the central axis of the femoral neck in the lateral view should be within the range of $160-180^{\circ}$ in both views to achieve acceptable reduction of the femoral neck fracture as previously described by Garden [11, 12].

Then through lateral approach the neck fracture was fixed by DHS with 2-hole side plate in 14 patients with additional derotation superior cannulated screw in 5 of them and by 3-4 cannulated cancellous lag screws in the remaining 3 patients, as shown in figure 2. In 1 patient with chest injury and intercostal tube inserted to the contralateral side of the fractures, the surgery was performed while the patient was in supine position on the orthopedic table from the start. After preliminary reduction and fixation of the shaft fracture by anteriorly placed DCP with two screws proximal and distal to the fracture, the necessary positioning of the lower limb to achieve anatomical reduction of the neck fracture was performed and DHS with a 14-hole side plate was inserted to fix both fractures. The other patient, to whom the same technique was applied, was a patient with fracture of the spine. In the last 5 patients of this series, iliac bone graft is added after stabilization of comminuted femoral shaft fractures.

\section{Follow-Up}

The average follow-up was 3.6 years (range 2-6 years). After surgery the patients were allowed to walk non-weight-bearing with crutches till a trace of callus started to appear at both fracture sites. Then the patients were permitted to gradually bear weight on the affected limb aided with crutches. The patient discarded crutches, once there was good bridging callus at femoral neck and shaft fractures. The patients then were seen for clinical and radiological assessment every 3 months during the first 2 years and every 6 months thereafter. The patients' data and their surgical treatment are shown in table 1 .

\section{Assessment of Outcome}

The primary outcome was assessed by fracture union as seen on serial radiographs and clinical function as estimated by Friedman and Wyman System [2]. The secondary outcome was assessment of the occurrence and rate of complications of the surgical injury such as deep infection requiring débridement, nonunion (3-5 months since the fracture without signs of progressive healing seen in serial radiographs with visible fracture line and/or absence of bridging callus), avascular post-traumatic osteonecrosis of the femoral head, malunion of the fracture and rate of revision surgery. The clinical outcome was assessed as good when there was no limitation of activities of daily living (ADL), no pain and less than $20 \%$ loss of hip or knee motion. A fair result indicates mild limitation of ADL, mild to moderate pain and $20-50 \%$ loss of motion. A poor 
Table 1. Patients' data

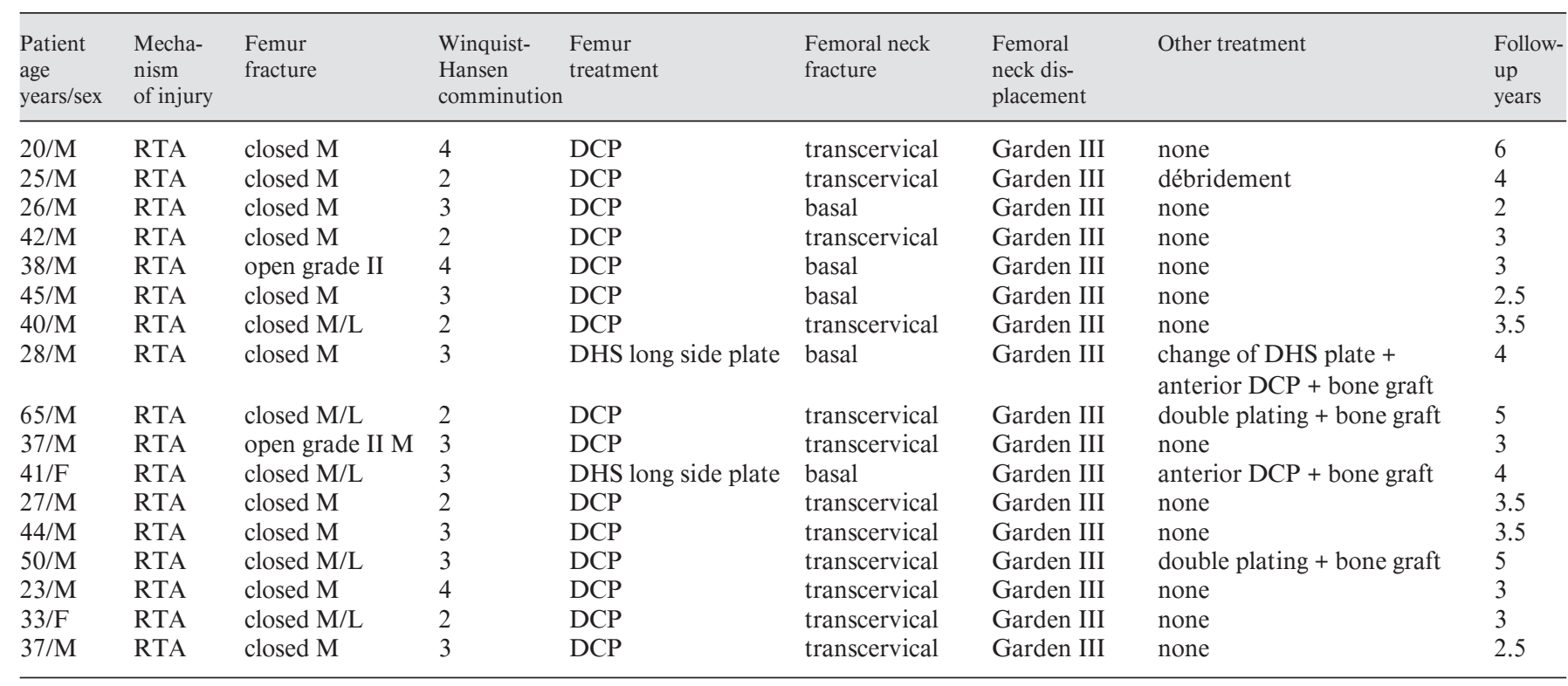

RTA = Road traffic accident; $\mathrm{M}=$ middle; $\mathrm{L}=$ lower.

result is associated with moderate limitation of ADL, severe pain, and more than $50 \%$ loss of motion.

\section{Statistical Analysis}

All patients' outcomes were analyzed according to the intentionto-treat principle. In order to express the long-term effectiveness of the operative treatment of ipsilateral femoral shaft and neck fracture using DCP, DHS and/or cannulated screw implants, relative risks were calculated with corresponding $95 \%$ confidence intervals. The relative risk expressed the proportion of patients with complaints (complications) related to the treatment strategy under evaluation.

\section{Results}

There was $100 \%$ union of the femoral neck fractures. In $15(88 \%)$ patients, the femoral neck fracture healed in an anatomic position in an average time of 4 months (range 2.5-5 months). In $2(12 \%)$ patients the femoral neck fracture united in $10^{\circ}$ varus angulation. No osteonecrosis of the femoral head was noticed in any patient till the last follow-up. Thirteen (77\%) of the 17 shaft fractures healed in an average of 5.4 months (range 4-9 months), as shown in figure 3 . Four (23\%) patients had nonunion and metal failures. Two patients in whom DHS with long side plate was utilized to fix both fractures were subjected to a revision surgery. In 1 patient the revision surgery was done 4 months after the index surgery due to nonunion and backing out of the side plate and screws and collapse of the femoral shaft reduction and fixation. In the other patient, the fracture failed to unite 5 months after index surgery with impending metal failure (bending of the DHS long side plate). On an ordinary surgical table, in supine position, the first patient was operated upon by changing the DHS side plate into a longer one after reducing the shaft fracture into nearly anatomical alignment. The fixation was augmented by another anterior 8-hole-broad DCP. Iliac bone graft was packed around the nonunion site. The same technique was applied in the other patient but without changing the DHS plate, which we found well anchored to the femoral shaft with rigid and adequate screw fixation as shown in figure 4 . In both patients the diaphyseal femoral fractures united 5 and 5.5 months after revision surgery, respectively. In another 2 patients (the oldest in the group with 50 and 65 years of age, respectively), the femoral shaft fixation DCP failed with backing out of the plate and screws with no trace of union after 2 months in the first patient and 2.5 months in the second. The 2 patients were operated upon by revision surgery where two anterior and longer lateral DCP plates were applied together with bone graft. The two complicated fractures united 6 and 8 months, respectively, following the revision surgery. It was proved later that 
Fig. 3 a, b. Healing of both fractures of the femoral neck and shaft in anatomical alignment after fixation by DHS and DCP.
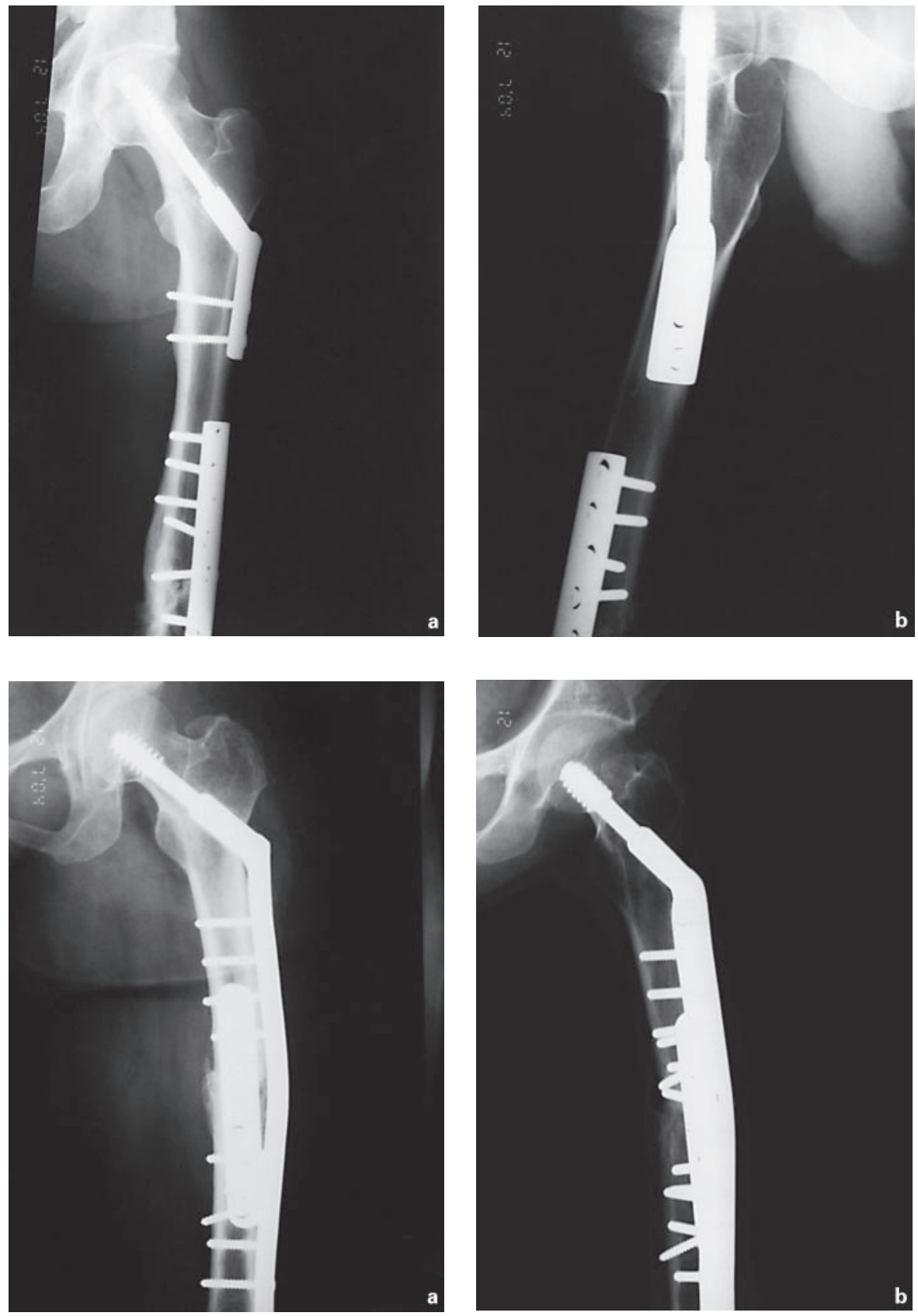

Fig. 4 a, b. Healing of both fractures of the femoral neck and shaft after the revision surgery of bone graft and anterior DCP because of metal failure and nonunion of the femoral shaft fracture. and the wound healed within 3 weeks of the second surgery. The overall clinical results of the patients as assessed according to Friedman and Wyman [2] showed that 13 patients (77\%) had good rating and 4 patients (23\%) fair rating. After the revision surgery $15(88 \%)$ patients had good outcome and only $2(12 \%)$ patients had fair clinical results. A summary of the results is shown in table 2 . 
Table 2. Results of surgical management of ipsilateral fractures of femoral neck and shaft

\begin{tabular}{llc}
\hline Results of femoral neck fracture fixation & Results of femoral shaft fracture fixation & Overall clinical outcome \\
\hline Union $(\mathrm{n}=17) 100 \%$ & Union $(\mathrm{n}=13) 77 \%$ & $\begin{array}{l}\text { With primary surgery } \\
(\mathrm{n}=13) 77 \% \text { good }\end{array}$ \\
Time of union & Time of union & $(\mathrm{n}=4) 23 \%$ fair \\
$\quad$ Average 4 months & Average 5.4 months & \\
$\quad$ Range $2.5-5$ months & Range $4-9$ months & With revision surgery \\
Varus malunion $(\mathrm{n}=2) 12 \%$ & Metal failure $(\mathrm{n}=4) 23 \%$ with nonunion & $(\mathrm{n}=15) 88 \%$ good \\
Osteonecrosis $(-)$ & Infection $(\mathrm{n}=1) 6 \%$ & $(\mathrm{n}=2) 12 \%$ fair \\
Metal failure $(-)$ & Revision surgery $(\mathrm{n}=4) 23 \%$ & \\
Infection $(-)$ & & \\
Revision surgery $(-)$ & & \\
\hline
\end{tabular}

\section{Discussion}

The ipsilateral femoral neck and shaft fractures combination injury is a treatment challenge $[16,17]$. The findings of this study show that fracture stabilization by DCP for femoral shaft fractures and DHS or cannulated screws for the femoral neck fractures is an adequate surgical management. It also shows that using one device to fix both fractures may increase the risk of metal fracture and nonunion. The weakness of this report is that it is a retrospective nonrandomized study of a small number of patients (only 17), the basic cause of which is the rarity of high velocity complex injuries. The small number of patients creates difficulties in the statistical data analysis of outcome. It also precludes subgrouping the patients into two categories to assess the difference between fixing the neck fracture by DHS or cannulated screws. The study strength is in the adequate follow-up of the patients that ranged from 2 to 6 years with an average of 3.6 years. This ensured reliability of the rate of avascular post-traumatic osteonecrosis of the femoral head that may occur up to 2-3 years after femoral neck fractures.

The conventional orthopedic understanding indicates that the femoral neck fracture is potentially the more problematic part of this pattern of injuries [10]. This is mostly because the complications of the femoral neck fractures (nonunion and avascular osteonecrosis of the femoral head) are more difficult to treat, especially in young patients, who represent the main and usual victims of this complex fracture of femoral neck and shaft. Accordingly, treatment of femoral neck fractures takes precedence over the shaft fracture in most of the literature reports $[5,7,10,13]$. To ensure the best chance for union, the neck fracture should be reduced anatomically and fixed rigidly by cannulated cancellous lag screws or by
DHS. The advantage of the DHS implant with its additional side plate is in providing a stable construct by transferring the deforming shearing force to the lateral cortex of the diaphysis. This can withstand more stress, especially in more vertical neck fractures (Pauwels' type III) and with the usual comminution of the posterior aspect of the neck, otherwise the fracture fixation may collapse with varus displacement, fracture gaping, metal failure and nonunion due to the relative lack of trabeculae in the neck, the thin cortex in the subtrochanteric region and the inherent instability of the fracture [10-12, 18].

However, Alho [19, 20] thought that femoral shaft fracture was the main determining factor in patients' overall outcome as also reported by Watson and Moed [10], who have shown that femoral shaft nonunion is more difficult to treat with some patients requiring more than one revision surgery to achieve union. The results of the current study with $77 \%$ union in the femoral shaft fractures and 100\% union in the femoral neck fractures confirm the results of Alho $[19,20]$ and Watson and Moed [10] and emphasize that both fractures should be tackled with equal degrees of seriousness, concern and care due to their drastic complications. Both must be anatomically reduced and rigidly fixed.

Optimal treatment of one fracture may interfere with that of the other; this makes deciding which fracture to be managed first controversial. Some investigators [10, 21] suggested that the proximal fracture of the neck of femur should be managed before the femoral shaft. The idea was to avoid the possibility of further displacement of the femoral neck fracture and the liability of occurrence of osteonecrosis of the femoral head. The observation of many authors $[6,9,11,15]$ confirms that further displacement of femoral neck fractures was frequently noted after interlocking nail insertion. In our standard 
protocol, the shaft fractures were stabilized first and followed by the neck fractures in 15 of our 17 patients. No further displacement of the neck fractures or difficulty of fracture neck reduction and fixation was noticed during surgery.

All the ipsilateral femoral diaphyseal fractures in this series were comminuted fractures, similar to the pattern reported by Shuler et al. [5].

\section{Conclusion}

Our results show that fixation of ipsilateral femoral neck and shaft fractures by DCP for the shaft fractures and DHS with short side plate or cannulated cancellous lag screws for the neck fractures is a reliable and adequate technique in achieving union in both fractures and reducing the rate of complications. It is also recommended to avoid stabilization of both fractures by one device as in using DHS with a long side plate.

\section{References}

-1 Delaney WM, Street DM: Fracture of femoral shaft with fracture of neck of same femur: treatment with medullary nail for shaft and Knowles pins for neck. J Int Coll Surg 1953;19:303312 .

-2 Friedman J, Wyman T: Ipsilateral hip and femoral shaft fractures. Clin Orthop Relat Res 1986;208:188-196.

-3 Casey J, Chapman W: Ipsilateral concomitant fractures of the hip and femoral shaft. J Bone Joint Surg 1979;61:503-509.

-4 Bennett S, Zinar M, Kigus J: Ipsilateral hip and femoral shaft fractures. Clin Orthop Relat Res 1993;296:168-177.

5 Shuler E, Gruen G, Ditano O, Riemer B: Ipsilateral proximal and shaft femoral fractures: spectrum of injury involving the femoral neck. Injury 1997;28:293-297.

6 Schatzker J, Barrington T: Fractures of the femoral neck associated with fractures of the same femoral shaft. Can J Surg 1968;11:297305.

$>7$ Wolinsky P, Johnson K: Ipsilateral femoral neck and shaft fractures. Clin Orthop Relat Res 1995;318:81-90.
8 Gills, Nagi O, Dhillon M: Ipsilateral fractures of femoral neck shaft. J Orthop Trauma 1990 4:293-298.

$\checkmark 9$ Bhandari M: Evidence-based orthopedic trauma working group: ipsilateral femoral neck and shaft fractures. J Orthop Trauma 2003;17: 138-140.

10 Watson J, Moed B: Ipsilateral femoral neck and shaft fractures: complications and their treatment. Clin Orthop Relat Res 2002;399: 78-86.

11 Garden S: Stability and union in subcapital fractures of the femur. J Bone Joint Surg Br 1964;46:630-641.

12 Garden S: Reduction and fixation of subcapital fractures of the femur. Orthop Clin North Am 1974;5:693-694.

13 Gustilo B, Anderson T: Prevention of infection in the treatment of one thousand and twentyfive open fractures of the long bones. J Bone Joint Surg Am 1976;67:19-28.

14 Winquist A, Hanson T: Comminuted fractures of the femoral shaft treated by intramedullary nailing. Orthop Clin North Am 1980;11:633642 .
15 Winquist A, Hansen T, Clawson K: Closed intramedullary nailing of femoral fractures: a report of 520 cases. J Bone Joint Surg Am 1984; 66:529-541.

16 Parfenchuck A, Carter W, Young R: Ipsilatera fractures of femoral neck and shaft. Orthop Rev 1993;22:356-363.

17 Wiss A, Sima W, Brien W: Ipsilateral fractures of the femoral neck and shaft. J Orthop Trauma 1992;6:159-166.

18 Pauwels F: Biomechanics of the Locomotor Apparatus. Translated by Maquet P, Furlong R. Berlin, Springer, 1980, pp 15-25.

19 Alho A: Concurrent ipsilateral fractures of the hip and femoral shaft: a meta-analysis of 659 cases. Acta Orthop Scand 1996;67:19-28.

$>20$ Alho A: Concurrent ipsilateral fractures of the hip and shaft of the femur: a systematic review of 722 cases. Ann Chir Gynaecol 1997;86:326336.

-21 Zettas JP, Zettas P: Ipsilateral fractures of the femoral neck and shaft. Clin Orthop Relat Res 1981;160:63-73. 